\title{
An analysis of the particulate flow in cold spray nozzles
}

\author{
M. Meyer and R. Lupoi \\ The University of Dublin, Trinity College, Department of Mechanical \& Manufacturing Engineering, \\ Parsons Building, Dublin 2, Ireland \\ Correspondence to: M. Meyer (meyerm@tcd.ie)
}

Received: 19 February 2015 - Revised: 28 May 2015 - Accepted: 25 June 2015 - Published: 11 August 2015

\begin{abstract}
Cold Spray is a novel technology for the application of coatings onto a variety of substrate materials. In this method, melting temperatures are not crossed and the bonding is realized by the acceleration of powder particles through a carrier gas in a converging-diverging nozzle and their high energy impact over a substrate material. The critical aspect of this technology is the acceleration process and the multiphase nature of it. Three different nozzle designs were experimented under constant conditions and their performance simulated using Computational Fluid Dynamics tools. The Deposition Efficiency was measured using titanium as feedstock material and it was shown that it decreases with the cross-sectional throat area of the nozzle. Computational results based on a one-way coupled multiphase approach did not agree with this observation, while more sophisticated modelling techniques with two-way couplings can partially provide high-quality outcomes, in agreement with experimental data.
\end{abstract}

\section{Introduction}

New required standards and tolerances come along with an increasing demand of enhanced surface properties, making a new generation of coating technologies necessary and capable of applying high quality layers of advanced materials ( $\mathrm{Li}$ and Yang, 2013) onto substrates of other metals or alloys.

An alternative to conventional deposition technologies, such as Laser Cladding (Partes and Sepold, 2008), Plasma and Flame Spray (Easter, 2008; Pawlowski, 1995) is Cold Spray (CS). This method is free of melting and therefore avoids the unwanted effects of those techniques which operate under high temperature levels (Kuroda et al., 2011). High pressure gas is accelerated in a converging-diverging supersonic nozzle to velocities in the order of $1000 \mathrm{~m} \mathrm{~s}^{-1}$. The coating material is injected as powder into the nozzle and accelerated by the gas flow. As the powder particles strike against a substrate placed at a distance from the nozzle exit, they deform plastically and bond with the substrate material.

The ratio of particle mass that is deposited successfully over the particle mass fed into the nozzle is called Deposition Efficiency (DE). It is evident that DE strongly depends on the impact velocity of the particles (Pattison, 2006). Despite the simple design and working principle, the flow char- acteristics are very complex, e.g. due to trans- and supersonic velocities, boundary layer instability, turbulence, and particularly the presence of multiple phases. The rapid change that the flow variables undergo from the inlet to the outlet of the nozzle is the most critical factor.

This complexity makes the nozzle dynamics sensitive to manufacturing inaccuracies (Pattison, 2006). In addition, numerical methods are in general not tailored for all the present local flow situations, e.g. the increased particle volume fraction in the throat region or the high pressure gradients in combination with extreme shear flow. Therefore, investigations often focus on specific aspects of the flow field independently.

For example, Lee et al. (2011) published a numerical investigation about the effects that gas operating conditions, particularly pressure and temperature, have on the flow field. Champagne et al. (2010) discussed the outcome of 1-Dnozzle calculation in comparison to 2-D axi-symmetric simulations and found measured velocities in an intermediate range between those theoretical approaches. A more application-related question was asked by Sova et al. (2013), who analysed the gas flow of a jet that impinges onto a mask. They tracked non-interacting particles that were released in the nozzle exit in order to find how they move through the 
mask. In contrast, Yin et al. (2011) observed at the impingement region how the impact velocity increases due to the change of the shock system as the substrate size is changed. A work by Park et al. (2010) concentrates on the gas phase as well, which is a good approximation, since it is dealt with nano-particles. Others, like Tang et al. (2014), were interested in numerical nozzle comparisons, but rather depending on the mixing conditions in the pre-chamber where the carrier and process gas streams join. Similarly, Yin et al. (2014) recently examined the effect of a variation of the injection pressure on the gas flow field on the one hand, but also on the dispersion of the particulate phase on the other. It was found, that the dispersion is strongly increased with enhanced injection pressure. It was also found by Sova et al. (2014) that the injection point as well has a significant effect on the particle dynamics, especially on the thermal conditions. Because the temperature has a major influence on the process efficiency, Li et al. (2011b) were engaged with the numerical modelling of heat transfer within the nozzle and substrate. In a similar manner, Zahiri et al. (2014) developed a 3-D-model that is also mainly concerned with the heat transfer between the nozzle, the fluid and the substrate. Interestingly, to point out the importance of turbulence treatment, a $k$ - $\varepsilon$-turbulence model resulted in over-predicted temperatures of the gas, which could be drastically improved by a model-calibration. Numerical works that focus on the particle behaviour were for example conducted by Li et al. (2011a), who were interested in the differences between under-expanded and overexpanded jets, using a variety of particle sizes. Suo et al. (2014) investigated recently how the particle velocity depends on the type of carrier gas, which was calculated with a simple particle tracking technique. The velocity error was of the order of $10 \%$ in this regard. Yin et al. (2009) again considered in a different context the interaction between particles during the deposition process, thus influencing the deformation of the particles upon impact, not considering any fluid dynamic effects.

Summarising, no studies so far deeply consider particleparticle interaction during the injection and acceleration, although the mixing conditions in the dense throat region and particle dispersion are found to be important in several studies. Several studies found the particle size to have important effects, even within not fully coupled phases, nevertheless, no investigation of the particle loading on the velocity distribution was conducted. There are no conclusive studies which link experimentally measured DE against nozzle design and their relationship at theoretical level.

This forms the starting point for the present study. In order to begin with the integration of all important modelling aspects, this work generates a connection between the particle loading and performance parameters, for example depending on different nozzle designs and operating conditions.

In this regard, experiments when depositing titanium onto aluminium tubes, are compared to numerically computed multiphase flows and discussed taking into account the fea-

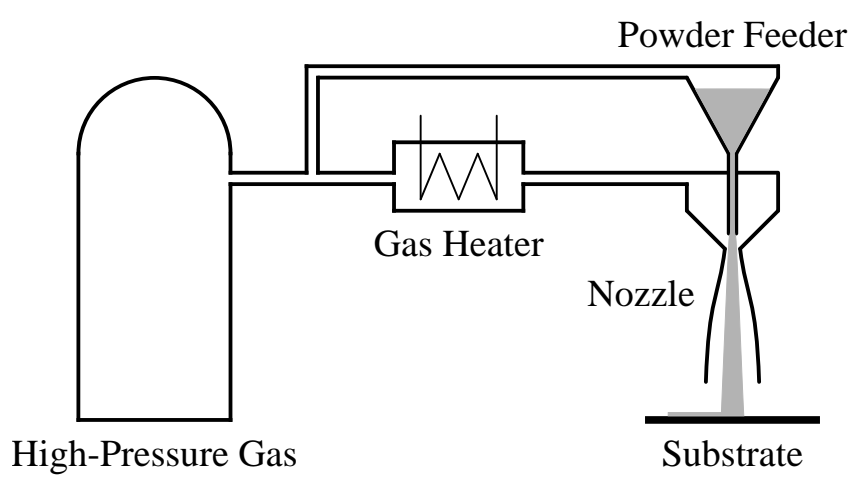

Figure 1. Set-up of the Cold Spray process.

tures of the most widely used numerical approach, the 1-waycoupled Lagrangian particle tracking. In addition, a more complex approach is discussed, a 2-way-coupled Lagrangian method with stochastic particle collisions, and applied to one of the nozzles, thereby comparing the outcomes at different particle loadings. It is found that conventional numerical methods can be inherently limited for the identification of the performance trends.

\section{Experimental procedure and results}

The general set-up for the CS process is shown in Fig. 1. The experiments were conducted utilizing a nitrogen type CS apparatus with an open loop powder feeder. The handling system was capable of delivering a working pressure of up to $3 \mathrm{MPa}$. A load cell read the powder mass flow rate, while a flow meter measured the gas flow rates in both the powder feeder line and the main line, where a gas heater was installed. This component was used to generate a higher inlet temperature, i.e. nozzle exit speed. Titanium powder (CPgrade $2,-45 \mu \mathrm{m}$ size, spherical) was injected in the subsonic region of the nozzle and deposited onto $50 \mathrm{~mm}$ diameter tubes ( $\mathrm{Al}$ 6082-T6) using three nozzles in order to assess their DE performance.

The geometrical details of the nozzles can be seen in Fig. 2. Correspondent values of the three designs (N1, N2, N3) are summarized in Table $1 . A_{\mathrm{i}}$ and $A_{\mathrm{e}}$ represent the inlet and exit cross-sectional area, respectively. $L_{\mathrm{c}}$ and $L_{\mathrm{d}}$ are the length of the converging and diverging sections of the nozzles and $A^{*}$ quantifies the cross-sectional throat area. For all test runs the same processing conditions were applied, i.e. the substrate was placed at a stand-off distance of $40 \mathrm{~mm}$ from the nozzle exit. The inlet pressure and temperature were set to $3 \mathrm{MPa}$ and $350{ }^{\circ} \mathrm{C}$ in order to reach the desired velocity regimes, the powder feed rate was measured to be $55 \pm 9 \mathrm{~g} \mathrm{~min}^{-1}$.

The measured feedstock powder mass flow enables the direct calculation of DE. The respective results are summarised in Table 2. Comparing N1 and N3, the DE of $16.3 \%$ is al- 


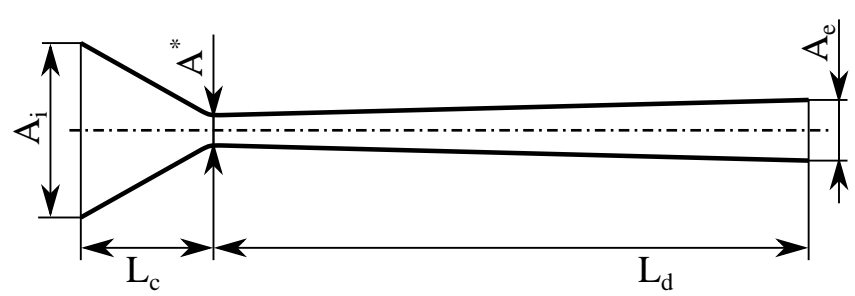

Figure 2. Geometry of the Cold Spray nozzle.

most doubled to a value of $33.3 \%$, despite the processing conditions remaining constant. Although their overall design is different, nozzle $\mathrm{N} 2$ and $\mathrm{N} 3$ exhibit similar DE values that correspond to the identical cross sectional throat area. A theoretical analysis was carried out so as to identify the key parameters to unravel the scientific reasons of the experimental outcomes.

\section{Uncoupled simulation}

In this section, a widely used approach is applied to all three nozzle geometries in order to survey its capabilities regarding an estimate of the experimentally detected behaviour. Therefore, the three cases were simulated with ANSYS Fluent v14.0. An initial analysis of this study was reported by Lupoi (2014).

The operating fluid nitrogen was set to be an ideal gas. The problem was reduced from a three dimensional to an 2-D-axi-symmetric flow. The Navier-Stokes equations for mass, momentum, and energy of the gas phase were solved for a steady state. Moreover, the equations were used in their Reynolds-averaged form and, consequently, extended by a $k$ $\varepsilon$-turbulence model with standard wall functions. The choice of this type of model was based on its common application in CS and the solution of the variables of interest experienced a negligible change when compared against non-equilibrium wall functions. Gravitational forces were considered negligible. The governing equations are according to FLUENT (2012):

$$
\begin{aligned}
\frac{\partial}{\partial x_{i}}\left(\rho u_{i}\right) & =0 \\
\frac{\partial}{\partial x_{i}}\left(\rho u_{i} u_{j}\right) & =-\frac{\partial P}{\partial x_{i}}+\frac{\partial \tau_{i j}}{\partial x_{i}} \\
\frac{\partial}{\partial x_{i}}\left(\rho e u_{i}\right) & =-\frac{\partial P u_{i}}{\partial x_{i}}+\frac{\partial\left(u_{j} \tau_{i j}-q_{i}\right)}{\partial x_{i}} \\
\frac{\partial}{\partial x_{i}}\left(\rho k u_{i}\right) & =\frac{\partial}{\partial x_{j}}\left[\left(\mu+\frac{\mu_{\mathrm{t}}}{\sigma_{\mathrm{k}}}\right) \frac{\partial k}{\partial x_{j}}\right]+G_{\mathrm{k}}+G_{\mathrm{b}}-\rho \varepsilon \\
& -Y_{\mathrm{M}}+S_{\mathrm{k}}
\end{aligned}
$$

Table 1. Geometrical details of the nozzles.

\begin{tabular}{llllll}
\hline Nozzle & $\begin{array}{l}A_{\mathrm{i}} \\
{\left[\mathrm{mm}^{2}\right]}\end{array}$ & $\begin{array}{l}L_{\mathrm{c}} \\
{[\mathrm{mm}]}\end{array}$ & $\begin{array}{l}A^{*} \\
{\left[\mathrm{~mm}^{2}\right]}\end{array}$ & $\begin{array}{l}L_{\mathrm{d}} \\
{[\mathrm{mm}]}\end{array}$ & $\begin{array}{l}A_{\mathrm{e}} \\
{\left[\mathrm{mm}^{2}\right]}\end{array}$ \\
\hline $\mathrm{N} 1$ & 314 & 30 & 3.1 & 180 & 28.3 \\
N2 & 44.2 & 15.5 & 5.7 & 190 & 47.8 \\
N3 & 314 & 20 & 5.7 & 190 & 47.8 \\
\hline
\end{tabular}

Table 2. Comparison of deposition efficiencies.

\begin{tabular}{cc}
\hline Nozzle & DE [\%] \\
\hline N1 & 16.3 \\
N2 & 32.5 \\
N3 & 33.3 \\
\hline
\end{tabular}

$$
\begin{aligned}
\frac{\partial}{\partial x_{i}}\left(\rho \varepsilon u_{i}\right) & =\frac{\partial}{\partial x_{j}}\left[\left(\mu+\frac{\mu_{\mathrm{t}}}{\sigma_{\varepsilon}}\right) \frac{\partial \varepsilon}{\partial x_{j}}\right]+C_{1 \varepsilon} \frac{\varepsilon}{k}\left(G_{\mathrm{k}}+C_{3 \varepsilon} G_{\mathrm{b}}\right) \\
& -C_{2 \varepsilon} \rho \frac{\varepsilon^{2}}{k}+S_{\varepsilon}
\end{aligned}
$$

In Eqs. (1)-(5), $\rho, u, P, e, \tau$, and $q$ denote the gas density, velocity, static pressure, internal energy, viscous stress tensor, and conductive heat flux. $k$ is the turbulent kinetic energy, $\varepsilon$ the eddy dissipation rate, while all other quantities are model-specific constants and source terms. Due to compressibility, a density-based solver was used with a second-order discretisation. The structured mesh was developed to suit the respective flow phenomena with a size of approx. $120000 \mathrm{el}-$ ements and tested to provide a mesh-independent solution for the gas phase. It was refined in the near-wall region to capture the boundary layer flow appropriately. The use of standard wall functions requests a wall-adjacent cell hight of no smaller than $y^{+}=15-30$ as it should not be placed in the viscous sub-layer. The flow variables, particularly the shear stress and the heat flux, tend to degrade otherwise. However, a sufficient number of grid points are required to resolve the boundary layer. Therefore, the mesh was designed for $y^{+}$ values between 20 and 80 . The throat radius was resolved with 110 points in the flow direction for a sufficient resolution of the flow gradients. Likewise the resolution at the nozzle exit was kept slightly refined in order to capture the shear layer of the jet. The cell size in the nozzle exit region was tested to be sufficiently fine to capture the shock pattern, i.e. with a change in solution due to mesh refinement less than $1 \%$. However, adaptive mesh refinement is an option for future work in order to optimise the shock resolution. This is particularly important for smaller particles $(\approx 1 \mu \mathrm{m})$ with shorter response times than in this study. Figure 3 shows the mesh at the nozzle inlet and throat as well as at the exit.

A pressure inlet boundary condition was applied to the nozzle inlet and set to the same values as in the experiments $\left(p_{0}=3 \mathrm{MPa}, T_{0}=350^{\circ} \mathrm{C}\right.$ ). The outlet pressure was defined 


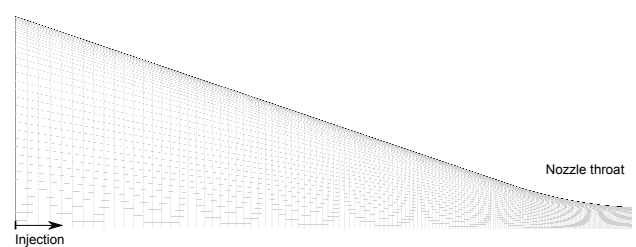

(a)

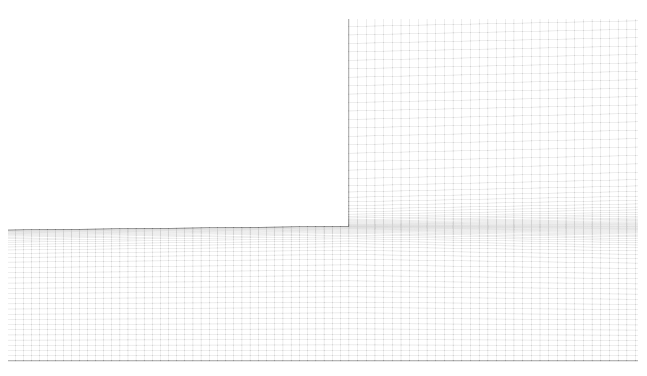

(b)

Figure 3. Computational mesh at the nozzle inlet, throat and exit.

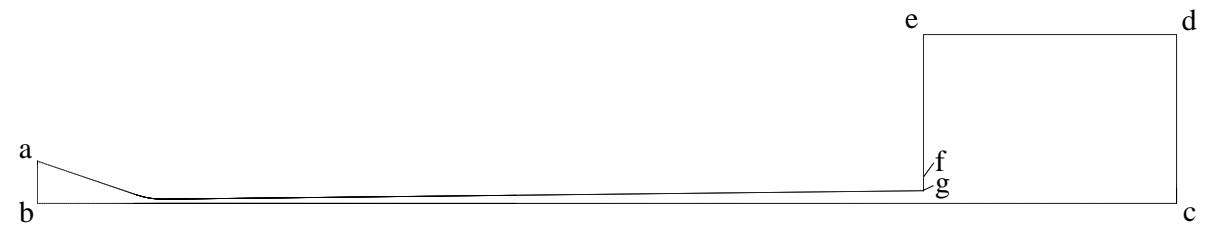

Figure 4. Geometry of computational domain and boundaries.

to be atmospheric pressure, sufficiently far downstream from the actual nozzle exit. An adiabatic no-slip boundary condition was applied to the nozzle wall. Figure 4 illustrates the computational domain and boundaries. Table 3 summarises the respective boundary conditions.

Concerning the particle phase modelling, a one-way coupled Lagrangian approach was chosen. In this respect, each particle ( $45 \mu \mathrm{m}$ diameter in the model) was released in the inlet zone and further described in a frame of reference that moves with the particle, solving the particle equations based on the local fluid properties. Nevertheless, the change of the gas state variables due to momentum and energy transfer to the particles is not taken into account, as it would require a two-way coupled multiphase model. This one-way coupling is often used in CS simulations, because it provided acceptable results under set conditions (Pattison et al., 2008). Mostly, it is claimed that this simplification is justified due to high Stokes numbers $S t$ and low momentum interaction parameters $\Pi_{\text {mom }}$ (Tabbara et al., 2010). In this manner, after obtaining a converged solution for the gas phase, particles are injected into the converging part of the nozzle. Their trajectories are calculated according to the force balance per unit particle mass given by Eq. (6).

$\frac{\mathrm{d} \boldsymbol{u}_{\mathrm{p}}}{\mathrm{d} t}=F_{\mathrm{D}}\left(\boldsymbol{u}-\boldsymbol{u}_{\mathrm{p}}\right)$

Here, the time differential is induced by the motion of the reference frame, but does not imply an unsteady tracking, since the solution is the same for every particle that is exposed to the flow at that specific position. $\boldsymbol{u}$ and $\boldsymbol{u}_{\mathrm{p}}$ are the local velocity vectors of fluid and particle respectively. $F_{\mathrm{D}}$ is a drag

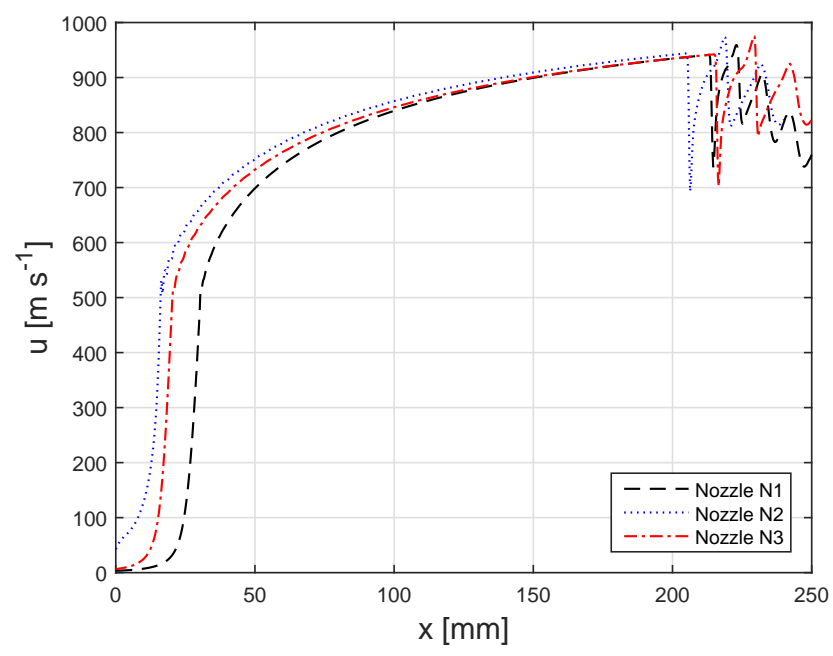

Figure 5. Comparison of nitrogen velocity profiles along the nozzle axis.

force term, that is based on the relative Reynolds number, see Eq. (7), with particle diameter $d_{\mathrm{p}}$.

$R e=\frac{\rho d_{\mathrm{p}}\left\|\boldsymbol{u}_{\mathrm{p}}-\boldsymbol{u}\right\|}{\mu}$

It can be seen, that the modelled acceleration of the particle is mainly influenced by the relative velocity and particle size.

Figure 5 presents the gas velocity profiles along the axial position for the different nozzle designs N1 to N3. The gas phase acceleration is most intense in the transonic region. Each profile shows the typical alternating pattern for over- 
Table 3. Boundary conditions for the axisymmetric calculation.

\begin{tabular}{lllll}
\hline Boundary & Condition type & $p_{0}$ & $\boldsymbol{v}$ & $T_{0}$ \\
\hline a-b & Pressure inlet & Specified & $\frac{\partial v}{\partial n}=0$ & Specified \\
b-c & Axial symmetry & $\frac{\partial p}{\partial r}=0$ & $\frac{\partial v}{\partial r}=0$ & $\frac{\partial T}{\partial r}=0$ \\
c-d, d-e, e-f & Pressure outlet & Specified & $\frac{\partial v}{\partial n}=0$ & $\frac{\partial T}{\partial n}=0$ \\
f-g, g-a & Adiabatic no-slip & $\frac{\partial p}{\partial n}=0$ & $\boldsymbol{v}=0$ & $\frac{\partial T}{\partial n}=0$ \\
\hline
\end{tabular}

expanded flows downstream of the nozzle. In all three nozzle types the gas reaches similar maximum values, although the acceleration in the transonic region differs.

Figure 6 shows a comparison of representative velocity profiles for single particle injections (at the nozzle centre line) in all nozzle design configurations. Since the accelerating drag force is directly related to the relative velocity of the fluid, it increases dramatically in the transonic region and reduces in the diverging section due to the fading gas expansion, as can be seen in Fig. 6. Since particle and gas speeds are still of different levels at the nozzle exits $A_{\mathrm{e}}$, some slight acceleration is maintained downstream of those points. The shock pattern does not significantly affect the $45 \mu \mathrm{m}$ particles because of their relatively high inertia. Interestingly, the all particles show very similar profiles and maximal velocities of approximately $595 \mathrm{~m} \mathrm{~s}^{-1}$ or $63 \%$ of the carrier gas speed.

Not only the simulated gas phase, but also the particulate material behaves in similar ways regardless of the considered design changes. However, in reality, the deposition performances are entirely different as reported in Table 2. Since the impact velocity is the main driver for DE as experimental conditions were not changed, this fundamental mismatch can only be explained through the fact that the modelling approach neglects important aspects of the process physics: it does not account for any gas-particle and particle-particle interactions. The phase coupling is therefore shown to play a more decisive role in CS nozzle dynamics.

\section{Coupled simulation}

If a significant fluid-particle interaction is present, it must have larger effects in N1 than in N2 and N3. The reason is a higher volume fraction of the particulate phase, originating from the smaller $A^{*}$ and a lower gas flow rate. A work published by Samareh and Dolatabadi (2008) provides this claim with further theoretical explanation. In this case, the inter-phase relations were modelled in a more sophisticated manner, using an Eulerian approach. Accordingly, both the fluid and the particulate phase were modelled as immiscible, interacting continua in the same reference frame. The authors showed a significant decrease in gas velocity at the exit due to the gain in momentum of the particulate phase as the loading was increased. This suggests significant interactions, at least on a theoretical level. A limit of this type of model is

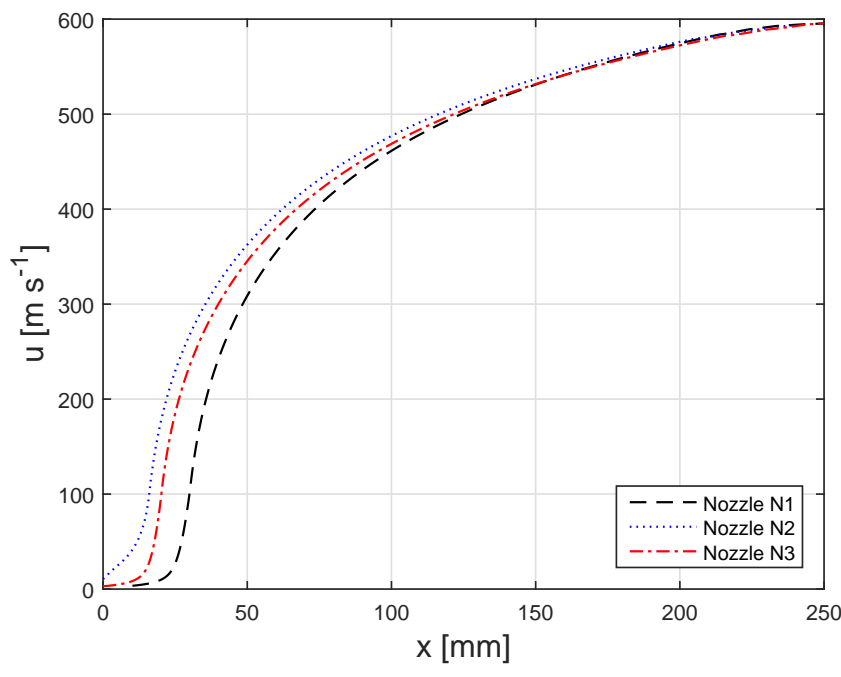

Figure 6. Comparison of titanium particle velocity profiles along the nozzle axis (uncoupled).

the dependency of its validity on relatively high particle density and uniform distribution.

The same authors contributed with another publication (Samareh et al., 2009) that is focused on the simulation of the shock pattern in the jet using a two-way coupled Lagrangian approach. It was found that flow patterns could be predicted with high accuracy, including effects of high particle loading in the jet. Using a particle size distribution of mostly small particles $(<10 \mu \mathrm{m})$, the calculated exit velocities were within the error range of the measurements. According to the authors, this agreement originated from the complex RSM turbulence model and the two-way phase coupling.

The latter approach is chosen in this study in order to compare different operating conditions. The inaccuracy of previously discussed models go back to the dependency of gas and particle dynamics on local particle loading and volume fraction. Therefore, the respective effects can be investigated if the only parameter that is changed is the particle feed rate, keeping the geometry constant. Since nozzle N1 is the design with the smallest restriction cross-section, it was chosen for this part of the study. It was investigated using the same gas flow conditions but varying particle feed rates from 0 to 16,32 , and through to $64 \mathrm{~g} \mathrm{~min}^{-1}$. The numerical mod- 


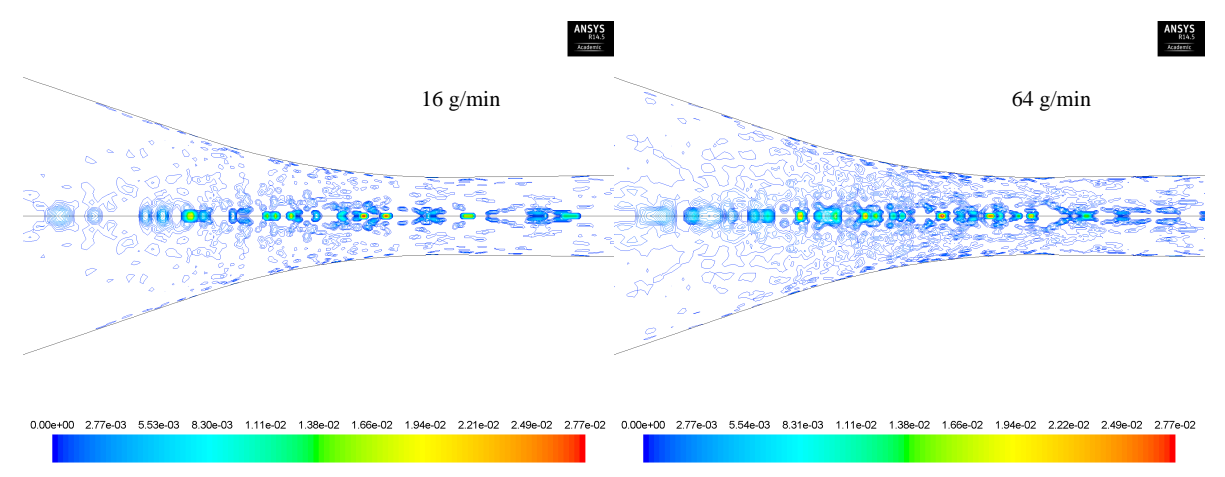

Figure 7. Volume fraction of particles in the throat region.

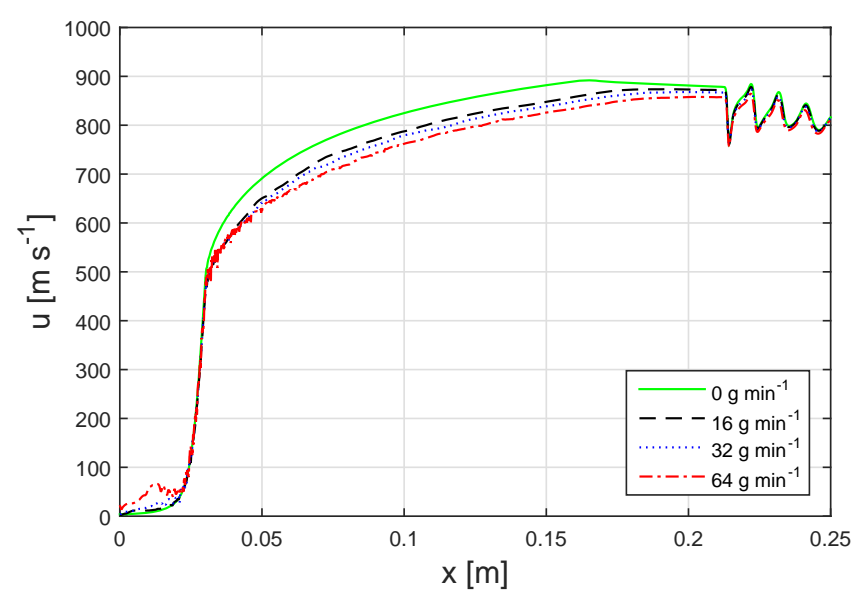

Figure 8. Comparison of nitrogen velocity profiles along axial position (coupled).

elling was adjusted as follows. The gas phase was modelled and solved as described above, but using a re-normalisation group (RNG) $k$ - $\varepsilon$-turbulence model as it amends the turbulent dissipation at different length scales and was successfully used in gas-particle flows (Fu et al., 2012). In terms of particle injection, apart from the feed rate no changes were made. Nonetheless, the two-way coupling corresponds to an unsteady tracking of particles and produces additional source terms in Eqs. (1)-(5). Likewise, the particle balance Eq. (6) is expanded by an additional force, that depends on the details of the coupling, including the concepts of virtual mass, turbulence coupling, Saffman lift, and stochastic particle-particle collisions, as well as a correction for high pressure gradients which plays a role in the vicinity of shock waves. Consequently, the solution requires an iteration for both the discrete and the continuous phase.

Figure 7 shows the volume fraction distribution in the throat region of nozzle $\mathrm{N} 1$ for the two loading cases of 16 and $64 \mathrm{~g} \mathrm{~min}^{-1}$. Local maximum values are as high as $2.8 \%$ and occur around the centreline, since less space is present for the gas phase. These maxima can be found more frequently in the case of higher loading, but the maximum par-

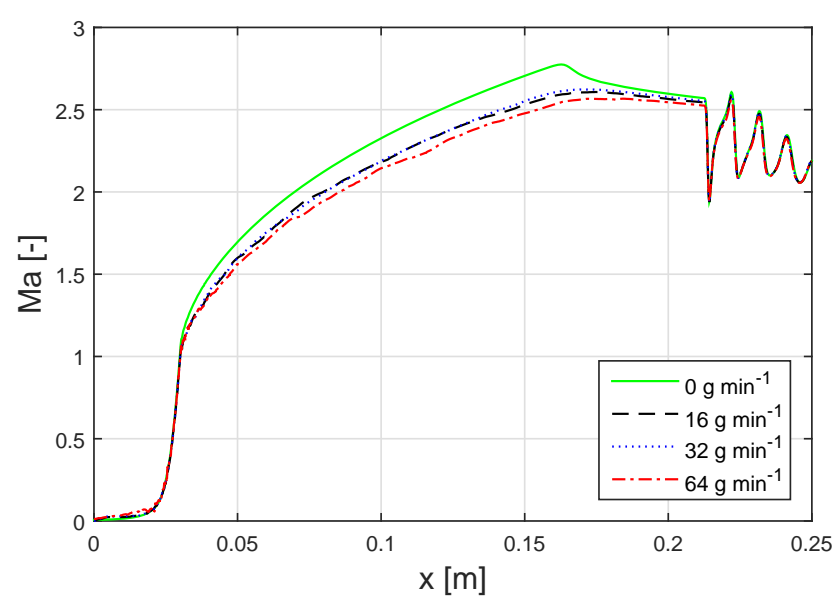

Figure 9. Comparison of gas Mach number along axial position (coupled).

ticle density does not increase measurably. Therefore, higher loadings apparently tend to cause the formation of lump-like spots of high volume fraction if the model accounts for particle particle-interactions. Nevertheless, the higher the particle volume fraction, the more likely collisions become. In this study, it is not observed how this evolution takes place with time while moving downstream through the flow field. This can cause some effect on the velocity observations and motivates a time-dependent solution. A time-averaging of the local particle behaviour could give a more general answer of its impact on the gas velocity.

Figures 8 and 9 show the velocity and Mach number profiles of nitrogen along the nozzle axis for all three particle feed rates and the unladen gas flow. It can be seen, that the velocity profiles are similar in the region of the throat and in the shock-expansion pattern of the jet. However, in the diverging section, the gas velocity decreases considerably with increasing particle feed rate, as more momentum is transferred to the discrete phase. Also, the Mach number profile shows a drastic reduction compared to the pure gas flow, although the differences between the three loading cases are 


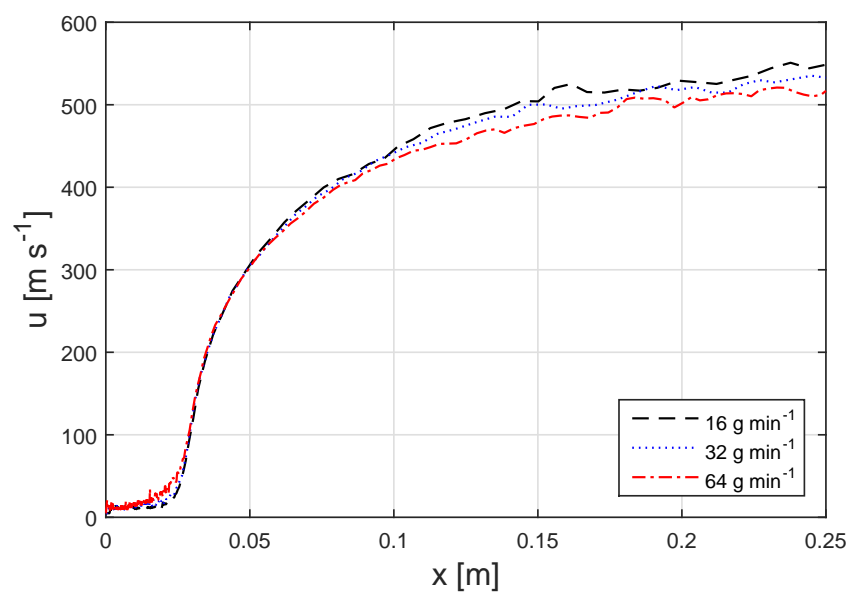

Figure 10. Comparison of titanium particle velocity profiles along axial position (coupled)

comparatively small. A Mach number drop within the last section of the divergent part of the nozzle can be observed for the pure gas flow, which diminishes with increasing feed rate. Interestingly, the gas velocity at the highest particle feed shows a different, more fluctuating trend as compared to the others, especially in regions of dense flow. These fluctuations indicate the importance of the turbulence coupling.

In Fig. 10 the analogous velocity profiles for the particles are shown. Here, the curve depicts the averages over intervals of 100 particles each considering an overlap of $40 \%$. In this manner, the respective data is reduced to a meaningful representation of the local velocity magnitude. As can be seen, the velocity profiles are similar in the throat region, while the final velocity in fact decreases with increasing feed rate by $8.9 \%$ for the present model. Velocities in the converging nozzle section appear to be the highest for the maximum feed rate which can be explained by a more dominant influence of particle-particle interactions that lead to local velocity maxima. It should be mentioned that the presented analysis neglects information about the velocity direction and hence the particle distribution.

The radial gas velocity profiles for three different axial positions are shown in Fig. 11. The first location with $x=30 \mathrm{~mm}$ corresponds to the nozzle throat, $x=100 \mathrm{~mm}$ is a central position of the diverging section and finally $x=211 \mathrm{~mm}$ is just downstream of the nozzle exit. For this comparison, the radial position is normalised by the local internal nozzle radius. It can be seen how the velocity profiles evolve from an accelerating flow characterised by boundary layers to a fully developed flow. In shock dominated flow at the nozzle exit, the pure carrier gas does not differ much from the loaded cases. However, the gas velocity reduces particularly in the vicinity of the centreline inside the nozzle. The figure shows that increasing the loading does not affect the gas phase as much as injecting $16 \mathrm{~g} \mathrm{~min}^{-1}$ titanium in the first place.

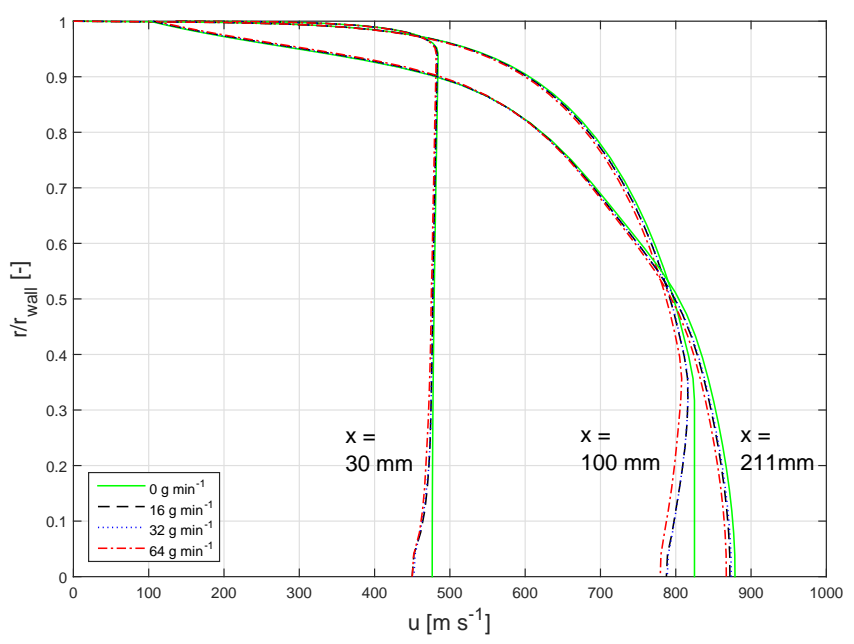

Figure 11. Comparison of gas velocity profiles along radial position (coupled)

Figures 12 and 13 compare the according radial particle velocity distributions at the nozzle throat and nozzle exit respectively. The different loading cases are compared against each other and represented by both the individual particles in the vicinity of the axial position and a second order polynomial fit. The first set, corresponding to the nozzle throat, shows homogeneously distributed particles for all cases, which agrees with the gas velocity profile. Because of the higher collision rate, the velocity level spreads out with increasing loading and the mean velocity raises. At the nozzle exit, the particles at $16 \mathrm{~g} \mathrm{~min}^{-1}$ exhibit a strong accumulation around the centreline. For the medium loading, this accumulation can still be seen, but the dispersion and the number of low speed particles have increased measurably. At $64 \mathrm{~g} \mathrm{~min}^{-1}$, the particles are spread over all radii with a nearto-constant velocity range, which is slightly lower than in the more dilute cases. The coupled model hence shows an important effect of mass loading on the particle dynamics. These are results at an instant of time, therefore the time-averaging of particle dynamics could possibly show the effects more clearly.

It is difficult to compare these results to the experimental data in default of directly measured velocities. In particular, a model to link the calculated velocities to DE is not available yet, because of a vast amount of practical influences. However, an attempt can be made to compare the calculated changes in velocity to the measured changes in $\mathrm{DE}$ as follows. Reducing the cross-sectional throat area of N3 to $\mathrm{N} 1$ by half, causes an increase in particulate loading. This is analogous to doubling the particle feed rate in N1 from 32 to $64 \mathrm{~g} \mathrm{~min}^{-1}$. The simulated particle velocity at the substrate stand-off distance $(x=0.25 \mathrm{~m})$ consequently drops by $4.4 \%$ to $511.9 \mathrm{~ms}^{-1}$. According to Champagne (2007), the $\mathrm{DE}$ for aluminium particles in heated air can experience a $65 \%$ decrease, as the particle velocity is reduced by $9.21 \%$ 

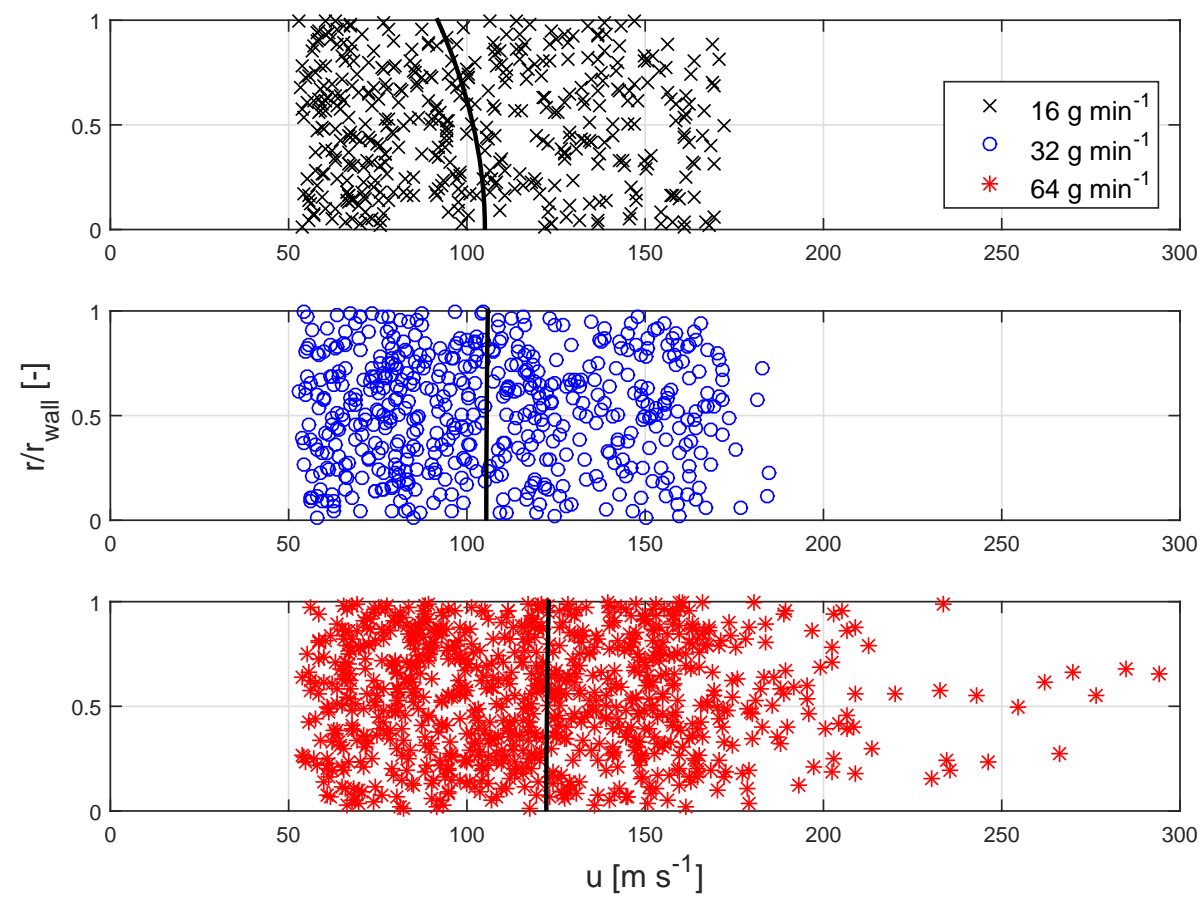

Figure 12. Comparison of titanium particle velocity profiles along radial position at the nozzle throat (coupled)
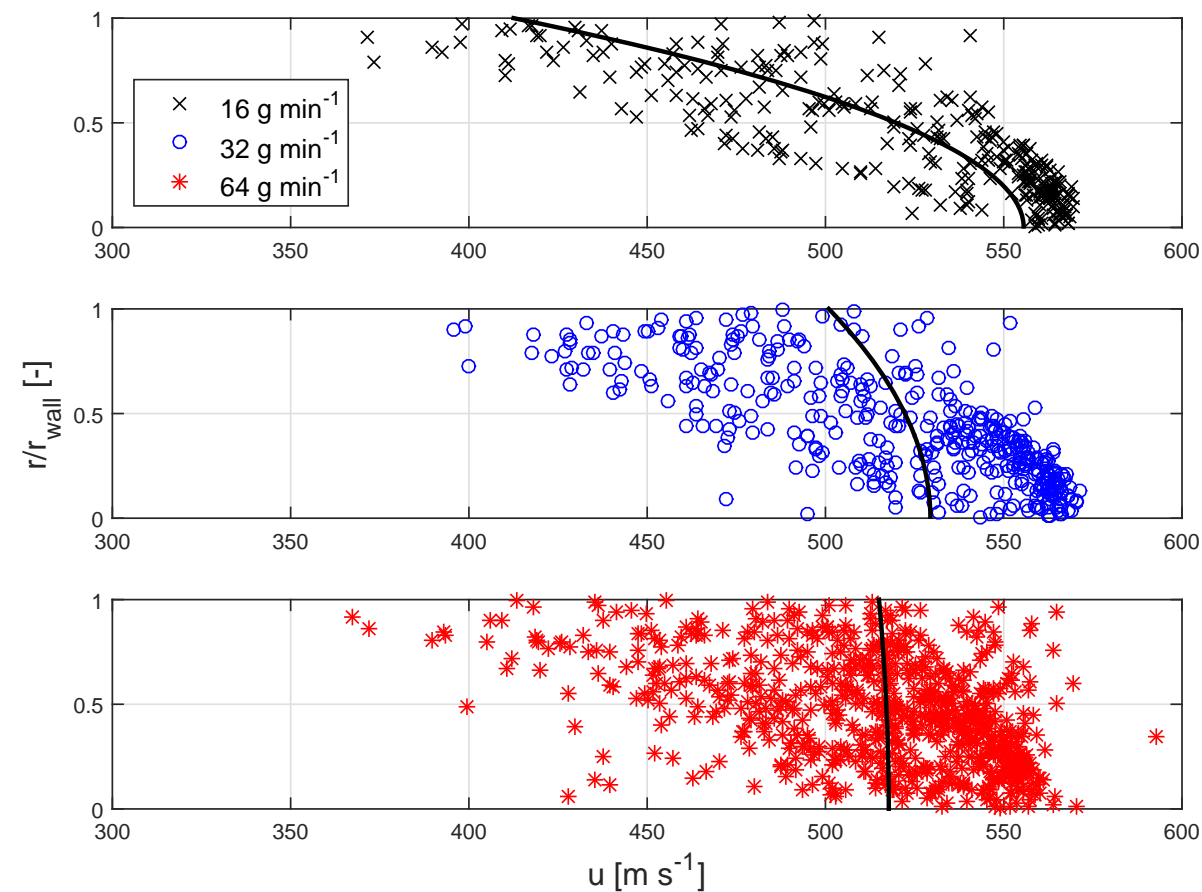

Figure 13. Comparison of titanium particle velocity profiles along radial position at the nozzle exit (coupled)

to $505 \mathrm{~ms}^{-1}$. Transferring this trend to the corresponding simulation results, the $4.4 \%$ decrease in particle velocity can be roughly estimated to cause a drop in DE of $31.1 \%$. As described in previous sections, the actual reduction of DE is as high as $51.05 \%$. Therefore, these results show a plausible tendency, however the magnitude is rather underestimated. A reason is the use of data from experiments with a different material and unequal parameters in this argumentation. The enhanced modelling is nonetheless a major improvement in 
face of the uncoupled Lagrangian simulation, where the decrease in DE was predicted to be $0 \%$.

However, in a work by Tabbara et al. (2010) the particle velocities were still considerably overestimated in a validation against experimental data despite a two-way coupling. It was reasoned mainly that this was due to the limits of the $k-\varepsilon$-turbulence model, although the realizable formulation was used that has improved performance for the jet spreading rate. However, particularly the dispersion of the particles was not captured sufficiently. Additionally, Han et al. (2009) showed that the deposition strongly depends on the particle injection process. These aspects represent another direct link between the geometry and the deposition efficiency. Taking the findings of the present study into account, the validity of more elaborately coupled modelling is therefore not only a question of design, gas operating conditions and particle feed rate, but also of local conditions, such as turbulence and particle distribution. This makes further development of more advanced methods and their validation necessary. It could be suggested to make use of a Reynolds-stress model (RSM) that was successfully applied in CS applications in good agreement with experimental observations (Samareh et al., 2009).

\section{Conclusions}

In this work, the deposition performances of three different De Laval nozzle designs under constant process conditions were investigated and explained by comparing them to numerical results. Titanium was deposited onto aluminium 6082-T6 tubes. It was found that the N1 nozzle, with the smallest throat cross-sectional area, performs the worst in terms of DE. Numerical simulations were performed based on fluid dynamic observations, using steady axisymmetric equations with a $k$ - $\varepsilon$-turbulence model and a one-way coupled discrete phase model. The computed results showed very similar velocity profiles for both phases in all nozzles. The variations in nozzle performance were therefore not numerically reproducible.

The insufficiency of the inter-phase coupling was derived as the main reason, as the comparison with more sophisticated modelling in literature showed. Using a two-way coupled discrete phase model, the effect of increased particle feed rate and hence density on the velocity distributions of both phases was shown to be noticeable for nozzle N1. However, the large number of factors, in relation to the nozzle design, the extreme changes in velocity, and volume fraction makes overall theoretical predictions difficult. Another important factor is the turbulence model, which is derived as another reason for uncertainty. These initial studies will require further development stages in this regard to achieve full validation.
Acknowledgements. The authors wish to express their gratitude to FP7 - Marie Curie (project acronym: SSAM) for the valuable support in developing the work presented in this article.

Edited by: M. Cotterell

Reviewed by: R. Clarke and one anonymous referee

\section{References}

Champagne, V.: The cold spray materials deposition process, Woodhead Publishing Limited, Cambridge CB21 6AH, England, 2007.

Champagne, V. K., Helfritch, D. J., Dinavahi, S. P. G., and Leyman, P. F.: Theoretical and Experimental Particle Velocity in Cold Spray, J. Therm. Spray Techn., 20, 425-431, 2010.

Easter, G.: Thermal Spraying - Plasma, Arc and Flame Spray Technology, Wexford College Press, Palm Springs, CA, 2008.

FLUENT: FLUENT Manual, FLUENT Inc., Lebanon, NH, 2012.

Fu, Y., Wang, T., and Gu, C.: Experimental and numerical analyses of gas-solid-multiphase jet in cross-flow, Proceedings of the Institution of Mechanical Engineers, Part G: Journal of Aerospace Engineering, 227, 61-79, 2012.

Han, T., Gillispie, B. A., and Zhao, Z. B.: An Investigation on Powder Injection in the High-Pressure Cold Spray Process, J. Therm. Spray Techn., 18, 320-330, 2009.

Kuroda, S., Watanabe, M., Kim, K., and Katanoda, H.: Current status and future prospects on warm spray technology, J. Therm. Spray Techn., 20, 653-676, 2011.

Lee, M.-W., Park, J.-J., Kim, D.-Y., Yoon, S. S., Kim, H.-Y., James, S. C., Chandra, S., and Coyle, T.: Numerical Studies on the Effects of Stagnation Pressure and Temperature on Supersonic Flow Characteristics in Cold Spray Applications, J. Therm. Spray Techn., 20, 1085-1097, 2011.

Li, C.-J. and Yang, G.-J.: Relationships between feedstock structure, particle parameter, coating deposition, microstructure and properties for thermally sprayed conventional and nanostructured WC Co, Int. J. Refract. Met. H., 39, 2-17, 2013.

Li, S., Muddle, B., Jahedi, M., and Soria, J.: A Numerical Investigation of the Cold Spray Process Using Underexpanded and Overexpanded Jets, J. Therm. Spray Techn., 21, 108-120, 2011 a.

Li, W.-Y., Yin, S., Guo, X., Liao, H., Wang, X.-F., and Coddet, C.: An Investigation on Temperature Distribution Within the Substrate and Nozzle Wall in Cold Spraying by Numerical and Experimental Methods, J. Therm. Spray Techn., 21, 41-48, 2011 b.

Lupoi, R.: Current design and performance of cold spray nozzles: experimental and numerical observations on deposition efficiency and particle velocity, Surf. Eng., 30, 316-322, 2014.

Park, J.-J., Lee, M.-W., Yoon, S. S., Kim, H.-Y., James, S. C., Heister, S. D., Chandra, S., Yoon, W.-H., Park, D.-S., and Ryu, J.: Supersonic Nozzle Flow Simulations for Particle Coating Applications: Effects of Shockwaves, Nozzle Geometry, Ambient Pressure, and Substrate Location upon Flow Characteristics, J. Therm. Spray Techn., 20, 514-522, 2010.

Partes, K. and Sepold, G.: Modulation of power density distribution in time and space for high speed laser cladding, J. Mater. Process. Tech., 195, 27-33, 2008.

Pattison, J., Celotto, S., Khan, A., and O'Neill, W.: Standoff distance and bow shock phenomena in the Cold Spray process, Surf Coat. Tech., 202, 1443-1454, 2008. 
Pattison, J. A.: Cold Gas Dynamic Manufacturing, Ph.D. thesis, Darwin College, University of Cambridge, Cambridge (UK), 2006.

Pawlowski, L.: The Science and Engineering of Thermal Spray Coatings, Wiley, New York, NY, 1995.

Samareh, B. and Dolatabadi, A.: Dense Particulate Flow in a Cold Gas Dynamic Spray System, J. Fluid. Eng.-ASME, 130, 817021-81702-11, 2008.

Samareh, B., Stier, O., Lüthen, V., and Dolatabadi, A.: Assessment of CFD Modeling via Flow Visualization in Cold Spray Process, J. Therm. Spray Techn., 18, 934-943, 2009.

Sova, A., Doubenskaia, M., Grigoriev, S., Okunkova, A., and Smurov, I.: Parameters of the Gas-Powder Supersonic Jet in Cold Spraying Using a Mask, J. Therm. Spray Techn., 22, 551-556, 2013.

Sova, A., Grigoriev, S., Kochetkova, A., and Smurov, I.: Influence of powder injection point position on efficiency of powder preheating in cold spray: Numerical study, Surf. Coat. Tech., 242, 226-231, 2014.

Suo, X., Yin, S., Planche, M.-P., Liu, T., and Liao, H.: Strong effect of carrier gas species on particle velocity during cold spray processes, Surf. Coat. Tech., pp. 1-4, 2014.
Tabbara, H., Gu, S., McCartney, D. G., Price, T. S., and Shipway, P. H.: Study on Process Optimization of Cold Gas Spraying, J. Therm. Spray Techn., 20, 608-620, 2010.

Tang, W., Liu, J., Chen, Q., Zhang, X., and Chen, Z.: The effects of two gas flow streams with initial temperature and pressure differences in cold spraying nozzle, Surf. Coat. Tech., 240, 86-95, 2014.

Yin, S., Wang, X.-F., Li, W.-Y., and Xu, B.-P.: Numerical Investigation on Effects of Interactions Between Particles on Coating Formation in Cold Spraying, J. Therm. Spray Techn., 18, 686693, 2009.

Yin, S., Wang, X.-F., Li, W.-Y., and Li, Y.: Numerical Study on the Effect of Substrate Size on the Supersonic Jet Flow and Temperature Distribution Within the Substrate in Cold Spraying, J. Therm. Spray Techn., 21, 628-635, 2011.

Yin, S., Liu, Q., Liao, H., and Wang, X.: Effect of injection pressure on particle acceleration, dispersion and deposition in cold spray, Comp. Mater. Sci., 90, 7-15, 2014.

Zahiri, S. H., Phan, T. D., Masood, S. H., and Jahedi, M.: Development of Holistic Three-Dimensional Models for Cold Spray Supersonic Jet, J. Therm. Spray Techn., 2014. 